\title{
High maternal and under-five mortality in an ethnic minority predominant area in rural China: a mixed- methods study exploring mother and health- provider experiences
}

\section{Bonnie Odelia Wong}

University of California, Berkeley https://orcid.org/0000-0001-8363-8285

Junguo Xin

Sichuan University

Lihua Jiang

Sichuan University

Ruiou Wang

Sichuan University

Yi Liu

Sichuan University

\section{Xiao Ma}

Sichuan University

Li Zhao ( $\nabla$ zhaoli@scu.edu.cn )

Sichuan University https://orcid.org/0000-0002-6297-528X

\section{Research}

Keywords: Socio-cultural, Qualitative, China, Sichuan Province, Rural care, Health disparities, Maternal mortality, Maternal care utilization, Ethnic minority, Pregnancy, Birth

Posted Date: December 10th, 2019

DOI: https://doi.org/10.21203/rs.2.18405/v1

License: (c) (1) This work is licensed under a Creative Commons Attribution 4.0 International License. Read Full License 


\section{Abstract}

Background: China as a country met Millennium Development Goals regarding maternal mortality rate (MMR) and under 5 mortality rate (U5MR), but high MMR and U5MR persist in various counties, especially rural areas of Western China with predominantly ethnic-minority populations. This study aims to understand multiple factors impacting MMR and U5MR in Xide and Mianning counties in Liangshan Area of Sichuan Province with majority Yi ethnic minority population, as well as the experiences of healthcare providers and mothers in these counties.

Methods: Quantitative birth and mortality data from 2012 to 2014 were derived from public and hospital databases. Qualitative methods were used to identify factors which impact MMR and U5MR. We administered structured close-ended surveys to 60 mothers with neonates and 60 healthcare providers and administrators and conducted semi-structured interviews with 26 recently-pregnant mothers regarding their delivery and peri-natal care experiences and with 23 healthcare providers regarding health behaviors and factors driving health disparities.

Results: MMR in Xide (72.02 per 100,000) and Mianning (68.10) county are significantly higher than the average MMR for the overall Liangshan Area (43.41), Sichuan (24.40) or the national average (21.8). U5MR in Xide County (12.7\%) is consistently higher than in Mianning (7.29\%). Of 22 cases of maternal deaths occurring between 2012 and 2014, a disproportionate number (59.9\%) were non-hospital births, and $81.81 \%$ of those deaths were of Yi ethnic minority women. $73 \%$ of medical providers have less than a Bachelor degree and only $40.74 \%$ (Xide) or $68.52 \%$ (Mianning) scored $>80 \%$ on evaluations of obstetric and perinatal knowledge and skill. Only $57 \%$ of mothers were advised to take folic acid. Mothers who participated in prenatal care programs were more likely to have hospital deliveries $(p=0.033)$ and participate in post-natal care $(p=0.05)$. Significant barriers to care exist due to limited of resources, transportation, cost, health literacy, and cultural norms.

Conclusions: MMR and U5MR are higher in Xide and Mianning County and Yi women are disproportionately likely to die in childbirth. Policy changes are necessary to address these inequities, to increase access to quality medical care and improve knowledge and skill-based training for existing medical personnel.

\section{Plain English Summary}

Though China has greatly improved rates of maternal and childhood death, there are still significant differences between areas in China. The worst rates are in rural areas of Western China, including Liangshan Autonomous Area, with predominantly Yi ethnic minority people. Reasons for persistently high rates of maternal and childhood death in these remote areas include lack of information about pregnancy as well as lack of resources and trained healthcare workers in these areas to assist with deliveries and provide care. 
We collected data in two neighboring counties in Liangshan Area and found higher rates of maternal and childhood death in these counties compared to Liangshan, Sichuan Province, or China overall. We conducted interviews and collected questionnaires to understand what health services were provided, the quality of care provided, levels of medical knowledge amongst healthcare providers, and the experiences of mothers regarding their deliveries. We found that Xide county has a higher maternal and childhood death rate than Mianning County, and also had physicians with lower degrees of medical training and with less medical knowledge. Xide mothers were also less likely to receive healthcare before and after birth. We found that mothers who died in these two counties were more likely to be Yi ethnic minority, and to have delivered outside of a hospital. Our qualitative interviews show that there are complex reasons why mothers are not able to get quality healthcare and to deliver babies in hospitals, including cost, transportation, cultural norms, and health literacy.

\section{Background}

In 2000, the United Nations declared reducing childhood mortality (Under 5 Mortality Rate or U5MR) and maternal mortality rate (MMR) by $75 \%$ between 1990 and 2015 as components of their Millennium Development Goals (MDG) [1, 2]. Additionally, the World Health Organization (WHO) listed reduction of MMR to less than 70 deaths per 100,000 livebirths by 2030 as a significant component of their sustainable development goals (SDG) [3].

China met MDG goals regarding early childhood mortality, reducing U5MR from 54.1 per 1000 livebirths in 1990 to 12.5 per 100,000 live births in 2015 . Additionally, China met the targets for U5MR fastest, with 10.7 deaths per 1,000 livebirths in 2015 and met MDG 5, reducing MMR from 111.0 deaths per 100,000 livebirths in 1990 to 21.8 per 1000 live births in 2015 [4, 5, 6, 7]. However, significant heterogeneity regarding these mortality rates are found between provinces and counties [8] and many counties which still have maternal mortality ratios greater than SDG targets are in rural areas in western China [6].

This heterogeneity is rooted in differences in economic growth amidst different counties and provinces. Over the past several decades, China has undergone significant economic growth and increased investment in health, improving the overall health status of the Chinese population [9]. However, remarkable inequity exists in the socio-economic development between rural and urban areas, amidst different geographic regions and between ethnic and non-ethnic minority populations; this inequity has a significant impact on disparities in health care access and therefore on overall healthcare metrics of these populations: specifically, western China has a greater proportion of rural and ethnic minority residents, has a lower GDP, and a higher proportion of illiteracy [6].

Ethnic minorities in China have benefited from national poverty alleviation efforts, educational benefits, and less-restrictive family planning legislation allowing ethnic minority couples to have a second or third child under the one-child policy [10]. However, meta-analyses of inequities in ethnic minority health in Western China have revealed statistically significant higher maternal mortality, neonatal mortality, and 
infant mortality, and lower uses of antenatal care, health-care facility births, and child immunizations, even after controlling for factors such as wealth and education [11].

An analysis of preventable maternal mortality shows a higher ratio of preventable deaths in underdeveloped and in-land (non-coastal) regions. Significant factors associated with increased MMR in remote areas included both insufficient information about pregnancy and peri-natal health among women and out-of-date knowledge and skills of health professionals providing obstetric services [12]. Additional barriers for rural and ethnic-minority populations include transportation, family accommodation, cost, language or cultural differences, lack of confidence in biomedicine or discrepant views on childbirth, and limited trained personnel [13]. Attempts to improve MMR in rural areas have included encouraging hospital delivery despite difficulties with cost, quality of care, or transportation [14, $15,16]$, and local maternal and child hospital records are carefully scrutinized. Despite this fact, rural areas continue to have worse MMR and U5MR than their urban counterparts or the national average [6].

Liangshan autonomous prefecture is a remote area in Southern Sichuan Province, at the Sichuan-Yunnan border. In Liangshan, $51.7 \%$ of the population is Yi ethnic minority according to Public Health Bureau records. Earlier studies of Yi ethnic minority women described limited hospital births, with determinants of health care utilization including accessibility, quality of healthcare services, and concerns about cost, insurance, and over-use of drugs [17]. Transportation challenges have been shown to disproportionately affect ethnic minorities in Sichuan: ethnic minority regions have acute shortages of healthcare personnel, and limited transportation and access to technology [18].

This paper describes a mixed-method qualitative and quantitative project to understand maternal and early childhood mortality in a rural region of Sichuan Province, comparing MMR, U5MR, access to care, and provider and maternal experiences in two neighboring counties. Investigation of maternal and early childhood healthcare access and utilization is necessary to better develop health policies which advocate for the health of ethnic minority women and children.

\section{Methods}

\section{Study setting}

This study was conducted in Liangshan area, an autonomous region of seventeen counties with 5.1 million people in the southwest area of Sichuan Province. Liangshan area has the largest Yi ethnic minority population in China. Yi ethnic minorities are largely subsistence farmers who live in rural Sichuan, with limited access to education or health care [17]. While there has been improvement in the economic status and transportation infrastructure in the past few years, development has been unequal in its distribution. Even between neighboring counties, there are differences in access due to transportation and infrastructure. This study compares two neighboring counties, Xide County and Mianning County in the Liangshan area (Figure 1): a highway (G5) runs through Mianning County, which is comparatively more economically developed, but does not run through Xide County, which is less developed. 
FIGURE 1: Map of Xide and Mianning Counties

LEGEND: (A) Sichuan Province with circle indicating location of Liangshan Autonomous Area in southwestern corner of the province. (B). Liangshan Area with two circles in northern region indicating locations of neighboring Mianning and Xide Counties on either side of the G5 Highway. (C) G5 Highway runs through the eastern area of Mianning County, which borders Xide County. G5 Highway does not enter Xide County.

China's primary healthcare infrastructure includes both township health centers and village clinics in rural areas (see Figure 2), which are often staffed by a single health practitioner and the majority clinics are not equipped to provide basic blood or urine tests [19]. Urban areas have both community health centers and community health stations. Xide County has 24 township clinics and 120 village clinics; Mianning County has 32 township clinics and 227 village clinics.

FIGURE 2: China's Primary Healthcare System schema by Location

LEGEND: China's primary health system consists of community health stations and community health centers in urban areas, and village clinics and township health centers in rural areas. This simplified model shows that community health centers and township health centers serve both primary (blue) and secondary (orange) health care with basic hospital inpatient services. This diagram does not show the three-tiered, ten-class Chinese hospital system which is based on size of hospital, as well as specialization of medical, education, and training services provided. Arrows indicate that if patients are seen in a community health station which cannot adequately provide care, they can go directly to county or area-level hospitals rather than being transferred via community health centers.

\section{Study design}

This study combines quantitative data analysis with a qualitative approach to generate an in-depth understanding of the health status, healthcare access, and delivery experiences of mothers and children within the Liangshan Area.

Quantitative data collection included the collection of birth data and mortality data from Mianning and Xide County. This data was derived from a registration database jointly overseen by the Public Health Bureau and Department of Family Planning and augmented through collected data from relevant hospital administrators. This data includes the number of pregnant women, obstetric patients, planned hospital deliveries, unplanned hospital deliveries, home deliveries, as well as maternal deaths, neonatal deaths 
( $<28$ days), infant ( $<1$ year old) deaths, and deaths of children under five. Additional quantitative data regarding perinatal care was collected from five hospitals in Xide and Mianning County.

Qualitative data collection included four components: (1) structured, close-ended surveys for health management and healthcare personnel regarding healthcare provision of services including scope of service, level of training, and number of births and deaths in their care; (2) structured evaluations to determine medical knowledge of care providers); (3) structured, close-ended surveys for mothers with a child under the age of one with questions regarding demography, education, healthcare access, experience with past deliveries, prenatal care and postpartum care; and (4) semi-structured interviews with health administrators at county hospitals, health providers at county hospitals, community health centers and health stations, township clinics, and village clinics, as well as both focus groups and individual interviews with recently-pregnant mothers.

Structured, close-ended surveys examined the aggregate experience of healthcare providers and maternal patients in these two counties. Structured close-ended questionnaires allowed for minimization of interviewer bias, for statistical analysis of data, and for repetition of this study at later timepoints or in other geographic locations.

Semi-structured in-depth individual interviews were combined with focus group discussions to allow for triangulation of findings and exploration of cultural complexities and nuance. Interviews were conducted by Sichuan University trained research assistants in the local dialect. Interview guides were developed and pilot tested, adjusting questions and phrasing as necessary. Topics covered included experience with seeking maternal or peri-natal health, perception of medical needs specific to pregnancy and peri-natal care, cultural customs around birth, health access, and challenges in seeking healthcare.

\section{Sample selection and data collection}

Participants for the qualitative component of this study were purposively sampled to provide diverse responses regarding health status, healthcare access, and delivery experience for mothers and children in both Xide and Mianning Counties. Data was collected between August and September of 2015 and participants were selected as follows:

1. Provision of healthcare: A total of thirty frontline staffed were identified to fill out the survey on provisions of healthcare service and service scope. These thirty individuals were selected from five hospitals: the Liangshan State Maternity and Child Care Hospital (a state level tertiary care hospital located in Xichang City), two county level maternal and child hospitals: the Xide Maternal and Child Care Hospital and the Mianning Maternal and Child Care Hospital, and two general county hospitals: Xide People's Hospital and Mianning People's Hospital. From each of these five hospitals, 1-2 hospital directors or healthcare managers and 2-6 doctors or nurses in the departments of gynecology, obstetrics, or pediatrics were selected. 
2. Evaluation of Provider Knowledge and Skill: Questionnaires evaluating the knowledge and skill of medical providers were distributed to thirty doctors from county hospitals and township or village health centers. Seventeen providers were from the five county hospitals listed above and thirteen providers were from six randomly selected township health centers or village clinics in Xide and Mianning Counties. Thirty surveys were complete and valid, with a completion rate of $100 \%$.

3. Delivery Experience of Mothers: A total of sixty mothers with an infant under 1 year old completed questionnaires regarding their delivery experiences. Three village clinics were randomly selected from Xide and Mianning counties, at each village, the village doctor identified 5-7 mothers who fit selection criteria for interview. 21 mothers were interviewed in Xide village clinics and 18 mothers were interviewed in Mianning village clinics. Additionally, 11 mothers were interviewed while admitted at or seeking care at Xide county hospitals and 10 mothers were interviewed at Mianning county hospitals. All mothers were Yi ethnic minorities.

4. In-depth interviews and focus group discussions were conducted in parallel with surveys for hospital administrators, physicians, and mothers. Participants were selected from survey participants by convenience sampling: 7 in-depth interviews were conducted with county hospital administrators, 16 interviews were conducted with county and village physicians, and 26 interviews or focus groups were conducted with mothers. The research team conducted interviews at a convenient, quiet, and private location at county hospitals or village clinics.

After obtaining written consent from each participant, interviews and focus groups were conducted in the native language of the participants. Interviews and focus groups were conducted until saturation was reached and no further themes emerged from data. Information from these interviews and focus groups were handwritten in the field, transcribed into a word processing document, and coded by two researchers trained in qualitative research methods for thematic analysis via grounded theory.

\section{Inclusion and exclusion criteria}

Quantitative data was limited to that concerning the three years of interest (January 2012 to December 2014). Only surveys with complete answers were included. Inclusion criteria for mothers included those who had delivered a child in the past year and were over the age of 18. Exclusion criteria were mothers for whom their most recent childbirth was not in-line with the national policies regarding family planning. For most women, these policies were not applicable because of exceptions made for ethnic minority women. Many women for whom an additional child is against family planning legislation will choose to have the child in secret and avoid seeking maternal or perinatal health care procedures; these women were excluded from the study as these additional legal and social factors affected their health-seeking behavior and healthcare access. 
De-identified data was analyzed using SPSS version 23.0 (IBM, Chicago, IL, USA) and SAS Software (version 2011). Descriptive statistics (frequencies and percentages) were used to summarize numerical data. Differences between categorical variables were analyzed via two-sided Pearson chisquare tests. Analysis uses $95 \%$ confidence intervals and considers $p$-values $<0.05$ as significant.

\section{Results}

\section{Maternal and Child Mortality}

Health statistics data were calculated from basic birth data and analyzed for three consecutive years. In Sichuan province, the MMR was 24.40 out of 100,000 live births in 2014 year, while the Liangshan autonomous prefecture had an MMR of 43.41 out of 100,000 , significantly higher than both the province and national average. U5MR is similarly elevated in Liangshan in comparison to the urban Sichuan population $11.95 \%$ compared to 5.22\%). Both Xide and Mianning County have much higher MMR at 72.02 and 68.10 per 100,000. While both counties exhibit declining maternal mortality, U5MR, and neonatal mortality rates, Xide County has consistently almost double the U5MR and neonatal mortality than Mianning County (12.77\% vs $7.29 \%$ and 5.57 vs $2.87 \%$ ) (see Table $1)$.

TABLE 1:

Health Statistics for Liangshan Area, Xide County and Mianning County from 2012-2014

\begin{tabular}{llrrr} 
& & 2012 & 2013 & 2014 \\
\hline Liangshan Area & Population & $4,922,446$ & 5,018277 & $5,091,002$ \\
& U5MR (\%) & 14.6 & 11.74 & 11.23 \\
\hline Xide County & MMR (per 100,000) & 51.71 & 61.89 & 43.41 \\
\hline & Population & 215,213 & 220,861 & 226,709 \\
\hline & Living births & 1836 & 1707 & 1851 \\
\hline & Birth rate & 8.53 & 7.73 & 8.16 \\
\hline U5MR (\%) & 12.42 & 12.05 & 12.77 \\
\hline Mianning County & MMR (per 100,000) & 35.49 & 95.15 & 72.02 \\
& Population & 382,187 & 389,833 & 395,673 \\
& Living births & 7555 & 7778 & 6801 \\
\hline & Birth rate & 19.77 & 19.95 & 17.19 \\
& U5MR (\%) & 4.37 & 5.26 & 7.29 \\
\hline & MMR (per 100,000) & 36.42 & 36.63 & 68.1
\end{tabular}


Analysis of incidences of maternal mortality

Analysis of incidences of maternal mortalities which occurred in Liangshan Area during the years 2012 to 2014 show that maternal deaths are more common for Yi ethnic minority women, non-hospital deliveries, and non-standard prenatal examinations. Between 2012 and 2014, there were twenty-two cases of maternal deaths in the two counties of interest: 16 in Mianning and 6 in Xide. There were 22,420 total births in Mianning county and 8,790 births in Xide county.

A significantly higher proportion of maternal deaths (81.8\%) were of Yi ethnic minority women ( $p=0.03$ by Chi-square analysis), despite only $58.7 \%$ of women being of Yi ethnic minority. Of the 22 deaths, 5 women were not registered to receive prenatal care; of the 17 which were enrolled, 7 did not receive prenatal examinations at the hospital, and only 4 cases had more than three pre-natal visits. Of the 22 deaths, 9 cases occurred in the hospital, and 13 occurred at home or in transit to the hospital. Given that $78 \%$ of births in Xide and Mianning county combined occurred in hospitals, a significantly higher proportion of maternal deaths occur outside of the hospital ( $p<0.0001$ by Chi-square analysis). Among the cases of maternal death, there were high proportions of Yi ethnic minority women and women who delivered at home, and low proportions of women who received standard prenatal exams.

Status of Health Personnel education, training, and knowledge

Questionnaire data from health professionals in both counties reveal an overall low education background with 73.3\% of individuals possessing less than a Bachelor degree. Additionally, 33.33\% did not receive standardized medical training prior to practicing medicine (see Table 4). There are also discrepancies between Xide and Mianning Counties, as Xide county health providers have lower educational attainment and lower proportions of those who have received specialized medical training. Interviews corroborated this data, as most administrators noted that doctors in higher-level hospitals have more opportunities to receive specialized training in maternal and childhood health and that most trainings are located in county level hospitals. Administrators noted the inadequate number and training of doctors in these two counties, noting that most facilities were operating at $50 \%$ staff 
capacity and that less than half of Town Health Centers could carry out hospital deliveries. Both health administrators and village doctors noted that there is need for training of village doctors in basic maternal and child disease management.

TABLE 4: Characteristics of Healthcare Provider Participants

\begin{tabular}{|c|c|c|c|}
\hline & & Number $($ total $=30)$ & Percent (\%) \\
\hline \multirow[t]{2}{*}{ Gender } & Male & $\begin{array}{ll} \\
\end{array}$ & 10 \\
\hline & Female & 27 & 90 \\
\hline \multirow[t]{3}{*}{ Age } & $\square 30$ & 9 & 30.0 \\
\hline & $30-45$ & 17 & 56.7 \\
\hline & $\square 45$ & 4 & 13.3 \\
\hline \multirow[t]{3}{*}{ Highest degree } & Bachelor & 8 & 26.7 \\
\hline & Junior college & 15 & 50 \\
\hline & Technical college & 7 & 23.3 \\
\hline \multirow[t]{3}{*}{ Job title } & Doctor & 18 & 63.0 \\
\hline & Nurse & 9 & 33.3 \\
\hline & Village doctor & 1 & 3.7 \\
\hline \multirow[t]{3}{*}{ Years of employment } & $\square 10$ & 17 & 58.6 \\
\hline & $10-30$ & 10 & 34.5 \\
\hline & ૧30 & 2 & 6.9 \\
\hline \multirow[t]{2}{*}{ Place of employment } & County hospital & 17 & 56.7 \\
\hline & Township or village clinic & 13 & 43.3 \\
\hline \multirow[t]{2}{*}{ County } & Xide & 15 & 50 \\
\hline & Mianning & 15 & 50 \\
\hline
\end{tabular}

Based on the questionnaire which evaluated basic knowledge in categories regarding perinatal care and obstetric emergencies, only $40.74 \%$ of those tested scored over $80 \%$ in Xide and 68.52\% in Mianning, revealing a significant discrepancy in the knowledge and skill background between Xide and Mianning health care providers. This discrepancy can be seen regarding both general obstetric knowledge and perinatal care. Discrepancy in basic knowledge was also found between village and county doctors, as only 2 of 13 (15\%) village doctors scored above 80\% while 6 of 17 (35\%) county doctors scored above $80 \%$ (results not significant at $\mathrm{p}=0.14$ ).

Medical care access and availability for pregnant women

Perinatal care covers fetal ultrasounds, HIV/AIDS screening, folic acid counseling and distribution, and child vaccination. The majority of women receive their healthcare 
consulting from village doctors, who have established a high degree of trust with residents of villages. Based on data collected regarding the scope of service and participation in perinatal care programs in both Xide and Mianning County, significant discrepancy can be seen between the healthcare access and utilization of women in the two counties. Xide has lower rates of participation in pre-partum care, post-partum care, maternal and neonatal care; however, both Xide and Mianning County have high rates of high-risk maternal care (see Table 2). And though rising in both counties over the three study period years, Xide also has consistently lower rates of in-hospital births with $59.4 \%$ overall vs $85.3 \%$ in Mianning (see Table 3).

TABLE 2: Maternal and Peri-partum care participation rates by county over time

\begin{tabular}{r|llllllll} 
& \multicolumn{3}{c}{ Xide County } & \multicolumn{3}{c}{ Mianning County } \\
\cline { 2 - 9 } & 2012 & 2013 & 2014 & Average & 2012 & 2013 & 2014 & Average \\
Pre-partum care \% & 79.40 & 85.23 & 75.00 & $\mathbf{7 9 . 8 8}$ & 87.00 & 89.37 & 90.67 & $\mathbf{8 9 . 0 1}$ \\
Post-partum care \% & 64.05 & 85.40 & 74.80 & $\mathbf{7 4 . 7 5}$ & 88.15 & 89.52 & 90.28 & $\mathbf{8 9 . 3 2}$ \\
Maternal care \% & 60.04 & 85.23 & 70.48 & $\mathbf{7 1 . 9 2}$ & 82.89 & 83.73 & 86.67 & $\mathbf{8 4 . 4 3}$ \\
Neonatal care \% & 55.02 & 71.40 & 70.28 & $\mathbf{6 5 . 5 7}$ & 90.96 & 91.88 & 92.29 & $\mathbf{9 1 . 7 1}$ \\
High-risk maternal care & 100.00 & 100.00 & 100.00 & $\mathbf{1 0 0 . 0 0}$ & 96.45 & 95.68 & 99.23 & $\mathbf{9 7 . 1 2}$
\end{tabular}

TABLE 3: Hospital vs non-Hospital deliveries in Xide and Mianning Counties Hospital deliveries per year Non-Hospital deliveries per year

\begin{tabular}{lllllllll}
\hline Xide & 2012 & 2013 & 2014 & Overall & 2012 & 2013 & 2014 & Overall \\
(\%) & 1556 & 1904 & 1764 & 5224 & 1262 & 1249 & 1055 & 3566 \\
\hline Mianning & 55.21 & 60.38 & 62.57 & $\mathbf{5 9 . 4 2}$ & 44.79 & 39.43 & 37.43 & $\mathbf{4 0 . 5 8}$ \\
$(\%)$ & 8450 & 5201 & 19134 & 1367 & 13583 & 560 & 3286 \\
& 80.04 & 86.15 & 90.28 & $\mathbf{8 5 . 3 4 4}$ & 19.96 & 13.85 & 9.72 & $\mathbf{1 4 . 6 6}$
\end{tabular}

Based off of questionnaires from recently pregnant women in Xide and Mianning County, women in Mianning County are significantly more likely to participate in prenatal care, and to participate in neonatal and maternal health care programs than women in Xide County ( $\mathrm{p}<0.05$ by Chi Square analysis). Among all survey participants, 55 (91.7\%) participated in maternal care program. On average, most mothers initiate prenatal care at 3.5 months of gestation and have four prenatal care visits prior to delivery. Of the 60 mothers in our sample, 31 (57\%) were advised to take folic acid, and 18 (30\%) participated 
in maternal and/or neonatal care programs. By chi-square test analysis, age of the mother (age $>30$ ) is associated with higher participation in prenatal care programs, and participation in prenatal care programs is associated with hospital delivery $(p=0.03)$ and post-natal care program participation $(\mathrm{p}=0.05)$.

\section{Barriers to healthcare access}

From survey questionnaires and interviews with healthcare administrators, health providers, and recently-pregnant mothers, we identified the following five barriers to healthcare access:

1. Lack of resources: village doctors lack training, capacity, and resources to provide additional healthcare to pregnant women and children. Most village doctors do not have standalone medical clinics, but provide medical care in their own homes. Their equipment is limited to a stethoscope and blood pressure cuff. Most villages lack a scale, and all villages lack a dedicated refrigerator for vaccines and basic medications.

2. Transportation: recently pregnant women reported on questionnaires that they had mostly reached their healthcare destination by walking (33.3\%) or by car $(26.7 \%)$, with the remaining using public bus, motorcycles, or other transport. The average time spent in transit was 52 minutes and the longest was five hours. The location of village clinics is also important, as in villages with less developed road infrastructure, villagers must walk 5-8 hours to reach the village clinic. Mothers also cited the inconvenience of transportation due to few vehicles and poor road conditions as a major reason to have home deliveries.

3. Cost: Mothers noted in interviews and focus groups that the main reason for home births is economic challenges. Though the government has reduced the cost of hospital delivery and provides a healthcare subsidy of 500 RMB for mothers who deliver in a hospital, it is still less expensive to have a home delivery due to the costs of transportation to a hospital (a minimum 300 RMB for a chartered car) and additional expenses of being away from their village. Of note, pre-natal check-ups and child immunizations are freely provided. 
4. Health Literacy: Though the importance of folic acid is emphasized at each prenatal visit by village doctors, village doctors noted that a number of pregnant women worry that the drug negatively affects the fetus, and refuse the medication. One village doctor noted that "information cannot be easily communicated" to mothers due to low education levels. Mothers noted that amidst their peers, awareness of the importance of hospital delivery is not strong, and many do not know about government subsidies available for hospital deliveries.

5. Cultural Norms: As emphasized by all interviewees, within Yi ethnic minority culture, pregnancy is shameful. Therefore, many women hide their pregnancies until late in their pregnancy. This presents challenges for delivery of prenatal care, and man women cite this cultural stigma as a reason for not seeking pre-natal care or taking folic acid. Additionally, traditionally, Yi women deliver at home with only their motherin-law present and with only a pair of scissors to assist in the procedure.

\section{Discussion}

As noted by Gao, Zhou and Singh et al [6], progress in MMR in China has been unprecedented, largely due to the government's commitment to ensuring safe hospital births even in rural areas. However, there continue to be significant gaps between hospital delivery rates in urban and rural areas [20,21]. In general, births are concentrated in larger county hospitals with well-trained staff and higher resources, smaller township health centers focus on perinatal care and screening for high-risk pregnancies. This study shows evidence of significant heterogeneity between counties with regard to healthcare access and medical personnel education and training, and subsequently there is significant discrepancy in MMR and U5MR.

This study provides a case-study comparison between Xide and Mianning county in Liangshan Area and shows that Xide county, with higher MMR and higher U5MR, also has physicians with lower degrees of medical training who scored lower on a quantitative evaluation of basic perinatal and obstetric care knowledge. In correlation, Xide mothers were less likely to receive pre-partum, post-partum, maternal, or neonatal care.

Given these discrepancies in the training and qualification of health providers in these two counties, possible policy mechanisms for improving MMR and U5MR are to increase the number and quality of providers and to provide standardized training regarding maternal and peri-natal care. Increased numbers of health professionals are associated with reductions in U5MR, with largest associations seen in areas with slowly increasing GDP per capita [22]. The relatively poor quality of healthcare in western regions 
has already been attributed to the difference in numbers of health providers (3.7 million in the eastern region vs 2.2 million in the western region) $[6,23]$. Our research reveals a fundamental need to not only increase the number of healthcare personnel, but also to improve education and skill-based knowledge of existing healthcare providers in this area.

This research further shows that maternal deaths are significantly more likely to be non-hospital deliveries and that mothers who received pre-natal care were significantly more likely to have hospital deliveries and to receive post-natal care. This is in line with existing studies which show low utilization of peri-natal health (due to household, economic, systems factors) leading to higher MMR [24, 25, 26]. Thus, efforts should be taken to encourage hospital deliveries through increasing access to pre-natal care. The "Safe Motherhood" program implemented by the Chinese Ministry of Health and the UN Children's Fund (UNICEF) in Western China increased delivery rates by providing subsidies, improving project management, establishing obstetric technical guidance, strengthening the quality of care in township hospitals, and providing training to village doctors [27]. There has also been significant government investment and policy programs directed at these efforts per qualitative data in this research: first, a recent policy requires babies to be born in hospitals to receive birth registration and certificates, and second, enrolling all women into the rural cooperative insurance program to assure cost-free hospital delivery $[28,29,30]$.

However, as this research shows, despite government policies, there are significant barriers to healthcare access and hospital delivery. Our qualitative in-depth interviews reveal that despite government subsidies, many women are not aware of these subsidies and lack basic health-literacy to communicate with health providers. Thus, efforts should be taken at the village clinic level to educate women about available subsidies and the importance of hospital birth. Additionally, though transportation and access to hospitals is a previously researched factor for maternal mortality $[18,25,31]$, this research re-emphasizes the link between poverty and transportation effects, as well as how lack of emergency transport services and poor state of roads deter hospital deliveries.

Guo and Huang 2019 [8] note the challenge of looking at county level data due to the instability of birth rates considering the small size of county populations; qualitative data can be helpful to understand and assess factors rather than relying on quantitative data which may not be reliable. Our quantitative data showed that maternal deaths are disproportionately likely to be Yi ethnic minority women, confirming prior research that shows minority-prevalent counties have higher MMR than non-minority prevalent counties [32] and the need for further rsearch to understand this discrepancy [33]. The qualitative components of this research reveal crucial barriers to maternal health improvement including the need to address differences in cultural norms. Given the high MMR and U5MR in the Liangshan area, attention should be paid to working with Yi ethnic minority cultural practices and perspectives around pregnancy and childbirth. For example, as in the first trimester of pregnancy, strangers cannot know about a pregnancy, it is key to establish prenatal care in the early second trimester with Yi women, and also to provide widespread education for women of reproductive age to take prenatal vitamins and folic acid 
regardless of pregnancy status and to have access to up to date resources about first trimester prenatal care without traveling to a village clinic.

\section{Limitations}

Our study has several limitations. First, this study only looks at two counties and the results may not be easily applicable to other areas. Second, we did not conduct a complete or random sample of healthcare providers, conducting purposive sampling of providers. Thus, we cannot ensure that the providers who answered our quantitative knowledge and skills evaluation are a representative sample of healthcare providers in these counties. Third, we chose to compare MMR and U5MR in two counties which were purposely economically dissimilar rather than controlling for economic wealth and other factors which may impact MMR and U5MR. The difference in GDP between Mianning and Xide County is significant. Thus, we are unable to isolate possible confounding factors, and economic resources are a known compounding factor on healthcare access and resources. However, the purpose of this study was to compare two neighboring counties and to conduct an in-depth understanding of factors which may lead to differences in MMR and U5MR from provider and maternal patient perspectives. Lastly, due to the sensitivity of the issue and small number of mothers of dead infants, all mothers interviewed and surveyed were mothers who have live infants under one year old. The experiences of mothers with dead infants may be significantly different and provide additional understanding of factors which lead to infant mortality. Additionally, though researchers attempted to distance themselves from local health workers and healthcare providers, it is possible that women may have perceived interviewers as associated with healthcare workers which would impact their willingness to speak honestly during focus groups and interviews. We cannot conjecture how this impacts the reliability of our results.

\section{Conclusion}

This study provides an in-depth mixed-methods examination of maternal mortality in a specific region of interest in Western China. Liangshan Area is a rural area which is economically disadvantaged and with predominantly ethnic minority population. Our study provides a nuanced exploration of barriers to maternal healthcare which may contribute to high MMR and U5MR. Based on our research, further research directions and pilot interventions should address (1) the quality and training of existing healthcare personnel in rural areas, (2) education of women regarding importance of and avenues for hospital delivery, (3) improvements in transportation or knowledge regarding transportation access (4) culturally-competent methods of providing care alongside Yi customs stigmatizing pregnancy.

\section{Declarations}

Ethical approval: This study was reviewed and approved by the institutional review board (IRB) of West China Medical Centre at Sichuan University. All methods were performed in accordance with the relevant guidelines and regulations set by the ethics committee, and written informed consent was obtained all subjects prior to completing questionnaires. 
Consent for publication: Not applicable.

Availability of data and materials: The datasets used and analysed during the current study are available from the corresponding author on reasonable request. The transcripts generated and analyzed in the qualitative portion of this research are not publicly available due to the sensitivity of the data, but summaries of transcripts can be made available from the corresponding author on reasonable request.

Competing Interests: The authors declare that they have no competing interests.

Funding: This study was supported by Save the Children UK, China Programme: Promoting Essential Newborn Care in Liangshan Area (2015-2017) and the United Nations Children's Fund (2018-Nutrition2.1.2.3).

Authors' Contributions: LZ conceptualized the study; JGX, LHJ, ROW collected quantitative and qualitative data; JGX, BOW, and YL conducted data analysis. BOW wrote the manuscript. JGX, XM, and LZ provided additional review, editing of manuscript, and supervision of the study. All authors read and approved the final manuscript.

Acknowledgements. We are grateful for the local collaborators and study participants for their time and insights. We would like to thank Save the Children for their financial support of this project. We would also like to thank Ruikan Yang, Lin Zhang, Yao He, Yanran Li, Qian Zhou, Wenchong He, and Shuaitun Liang for their assistance with the data collection process. We also extend our thanks to Professors Min Yang and Zumin Shi for their feedback and assistance with analysis and writing of this manuscript.

\section{References}

[1] Goal 5: Improve Maternal Health. United Nations Millennium Development Goals. Accessed 15 July 2019. https://www.un.org/millenniumgoals/maternal.shtml.

[2] MDG5: Improve Maternal Health. World Health Organization. Accessed 10 July 2019. https://www.who.int/topics/millennium_development_goals/maternal_health/en/

[3] SDG3: Ensure healthy lives and promote wellbeing for all at all ages. World Health Organization Sustainable Development Goals. Accessed 15 July 2019. https://www.who.int/sdg/targets/en/. 
[4] UNICEF International Forum on Countdown to 2015 progress in maternal and child survival in China, United Nations International Children's Emergency Fund, Beijing. 2015. Accessed July 2019. http://www.unicef.cn/en/index.php?m=content\&c=index\&a=show\&catid=53\&id=4547

[5] Ministry of Foreign Affairs, People's Republic of China, United Nations System in China. Report on China's implementation of the Millennium Development Goals (2000-2015). July, 2015. https://www.fmprc.gov.cn/ mfa_eng/zxxx_662805/W020150730508595306242.pdf

[6] Gao Y, Zhou H, Singh NS, et al. Progress and challenges in maternal health in western China: a Countdown to 2015 national case study. Lancet Glob Health. 2017; 5: 3523-36.

[7] Liang J, Li X, Kang C, et al. Maternal mortality ratios in 2852 Chinese counties, 1996-2015, and achievement of Millennium Development Goal 5 in China: a subnational analysis of the Global Burden of Disease study 2016. Lancet. 2018; 393(10168):241-252.

[8] Guo Y, Huang YM. Realising equity in maternal health: China's successes and challenges. Lancet. 2019; 393:202-203.

[9] Report on China's Implementation of the Millenium Development Goals (2000-2015). Ministry of Foreign Affairs People Republic of China. United Nations System in China. July 2015.

[10] Hesketh T, Lu L, Xing ZW. The effect of China's one-child family policy after 25 years. N Engl J Med. 2005; 353: 1171-76.

[11] Huang Y, Shallcross D, Pi L, Tian F, Pan J, Ronsman C. Ethnicity and maternal and child health outcomes and service coverage in western China: a systematic review and meta-analysis. Lancet Global Health. 2017;6:e39-56.

[12] Liang J, Dai L, Zhu J, Li XH, Zeng WY, Wang H, Li Q, Li MR, Zhou R, Wang YP. Preventable maternal mortality: Geographic/rural-urban differences and associated factors from the population-based maternal mortality surveillance system in China. BMC Public Health. 2011; 11:243.

[13] Gyaltsen K, Gyal L, Gipson JD, Kyi T, Pebley AR. Reducing high maternal mortality rates in western China: a novel approach. Reproductive Health Matters. 2014; 22(44):164-173.

[14] People's Daily Online. 2002. Midwifery phased out in China's rural areas. 30 September. Online at: http://english.peopledaily.com.cn/.

[15] Feng XL, Guo SF, Hipgrave D, Zhu J, Zhang L, Song L, Yang Q, Guo Y, Ronsmans C. China's facilitybased birth strategy and neonatal mortality: a population-based epidemiological study. Lancet. 2011; 378(9801):1493-1500.

[16] Feng Xl, Xu L, Guo Y, Ronsmans C. Socioeconomic inequalities in hospital births in China between 1988 and 2008. Bulletin of the World Health Organization. 2011;89:432-441. 
[17] Harris A, Zhou Y, Liao H, Barclay L, Zeng W, Gao Y. Challenges to maternal health care utilization among ethnic minority women in a resource-poor region of Sichuan Province, China. Health Policy Plan. 2010; 25(4):311-8.

[18] Wang XL, Pan J. Assessing the disparity in spatial access to hospital care in ethnic minority region in Sichuan Province, China. BMC Health Services Research. 2016; 16:399-410.

[19] Li X, Lu JP, Hu S, Cheng KK, De Maeseneer J, Meng QY, Mossialos E, Xu DR, Yip W, Zhang HZ, Krumholz HM, Jiang LX, Hu SS. The primary health-care system in China. The Lancet. 2017; 390(10112):2584-2594.

[20] Long Q, Zhang T, Xu L, Tang S, Hemminki E. Utilisation of maternal health care in western rural China under a new rural health insurance system (New Co-operative Medical System). Trop Med Int Health $2010 ; 15: 1210-7$.

[21] Lu P, Zheng Q, Yu T: The investigative study on health care and serv ice of pregnancy and parturient in 5-provience Western area. Chinese Primary Health Care. 2000, 15: 648-650.

[22] Liang S, Macinko J, Yue DH, Meng QY. The impact of the health care workforce on under-five mortality in rural China. Human Resources for Health. 2019; 17:21.

[23] Han Y, Wei J, Song X, Sarah BJ, Wen C, Zheng X. Accessibility of Primary Health Care Workforce in Rural China. Asia Pacific Journal of Public Health. 2011; 24(5):833-847.

[24] Say L, Raine R. A systematic review of inequalities in the use of maternal health care in developing countries: examining the scale of the problem and the importance of context. Bull World Health Organ. 2007, 85 (10): 812-819.

[25] Gabrysch S, Campbell OM: Still too far to walk: Literature review of the determinants of delivery service use. BMC Pregnancy Childbirth. 2009, 9: 34-10.1186/1471-2393-9-34.

[26] Anson O. Utilization of maternal care in rural HeBei Province, the People's Republic of China: individual and structural characteristics, Health Policy. 2004; 70(2):197-206.

[27] Liu X, Yan H, Wang D. The evaluation of "Safe Motherhood" program on maternal care utilization in rural western China: a difference in difference approach. BMC Public Health. 2010; 10:566.

[28] Rokicki S, Donato K. Effects of China's Rural Insurance Scheme on Objective Measures of Health. Economics Working Papers. 20116; 16-01. Queen's Management School, Queen's University Belfast.

[29] Babiarz KS, Miller G, Yi HM, Zhang LX, Rozelle S. New evidence on the impact of China's New Rural Cooperative Medical Scheme and its implications for rural primary healthcare: multivariate difference-indifference analysis. Brit Med J. 2010;341. 
[30] Health and Family Planning Commission of Sichuan Province. Hospital delivery subsidy project in rural area. http://www.scwst.gov.cn/xx/xwdt/mtxw/201408/t20140806_3623.html. Accessed 20 Nov 2019.

[31] Atuoye KN, Dixon J, Rishworth A, Galaa SZ, Boamah SA, Luginaah I. Can she make it? Transportation barriers to accessing maternal and child health care services in rural Ghana. BMC Health Services Research. 2015; 15:133-143.

[32] Ren Y, Qian P, Duan Z, Zhao Z, Pan J, Yang M. Disparities in health system input between minority and non-minority counties and their effects on maternal mortality in Sichuan province of western China. BMC Public Health. 2017;17:750.

[33] Huang Y, Shallcross D, Pi L, Tian F, Pan J, Ronsmans C. Ethnicity and maternal and child health outcomes and service coverage in western China: a systematic review and meta-analysis. The Lancet Global Health. 2018;6(1)e39-356.

\section{Figures}
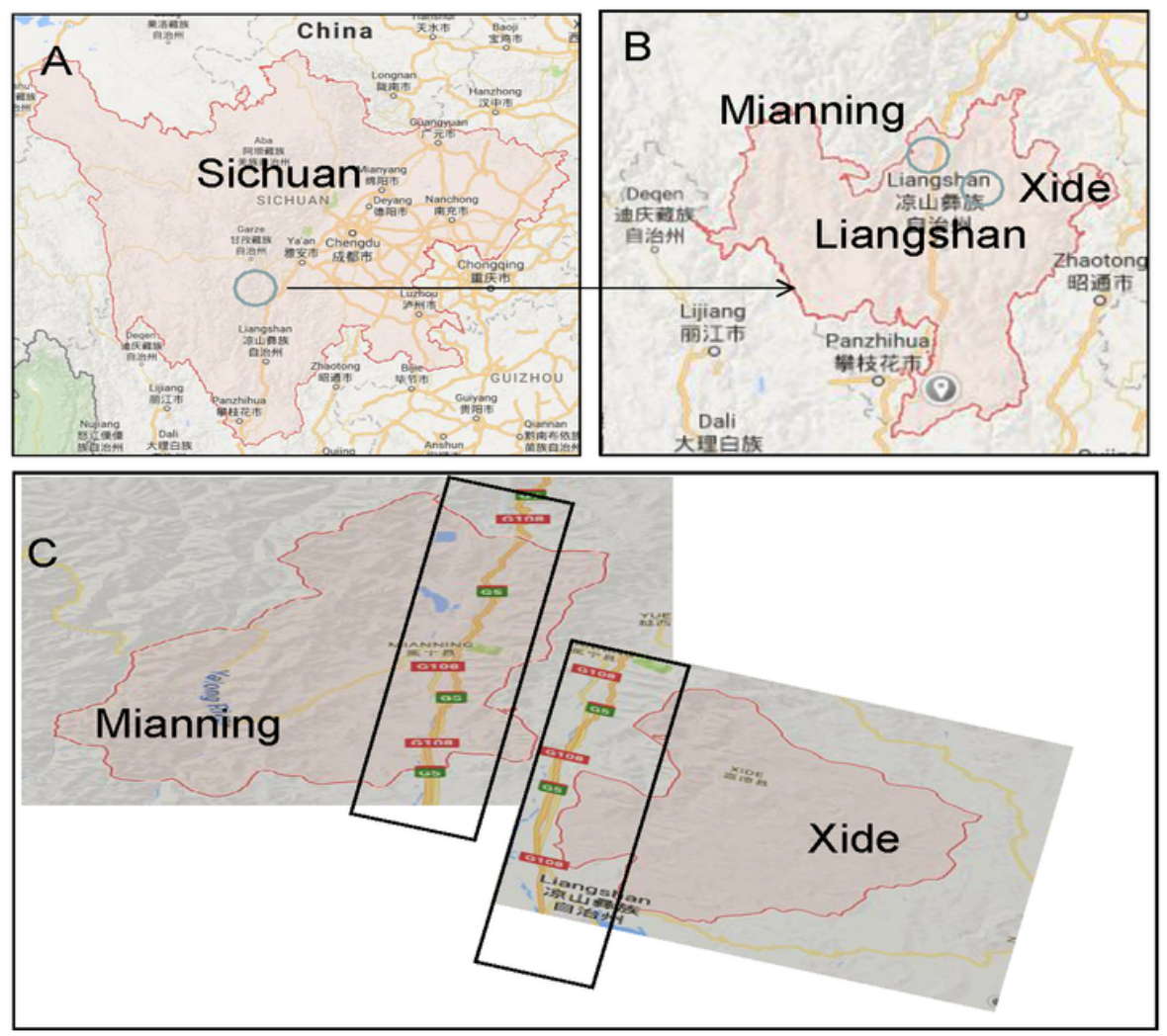

Figure 1 
Map of Xide and Mianning Counties LEGEND: (A) Sichuan Province with circle indicating location of Liangshan Autonomous Area in southwestern corner of the province. (B). Liangshan Area with two circles in northern region indicating locations of neighboring Mianning and Xide Counties on either side of the G5 Highway. (C) G5 Highway runs through the eastern area of Mianning County, which borders Xide County. G5 Highway does not enter Xide County. Note: The designations employed and the presentation of the material on this map do not imply the expression of any opinion whatsoever on the part of Research Square concerning the legal status of any country, territory, city or area or of its authorities, or concerning the delimitation of its frontiers or boundaries. This map has been provided by the authors.

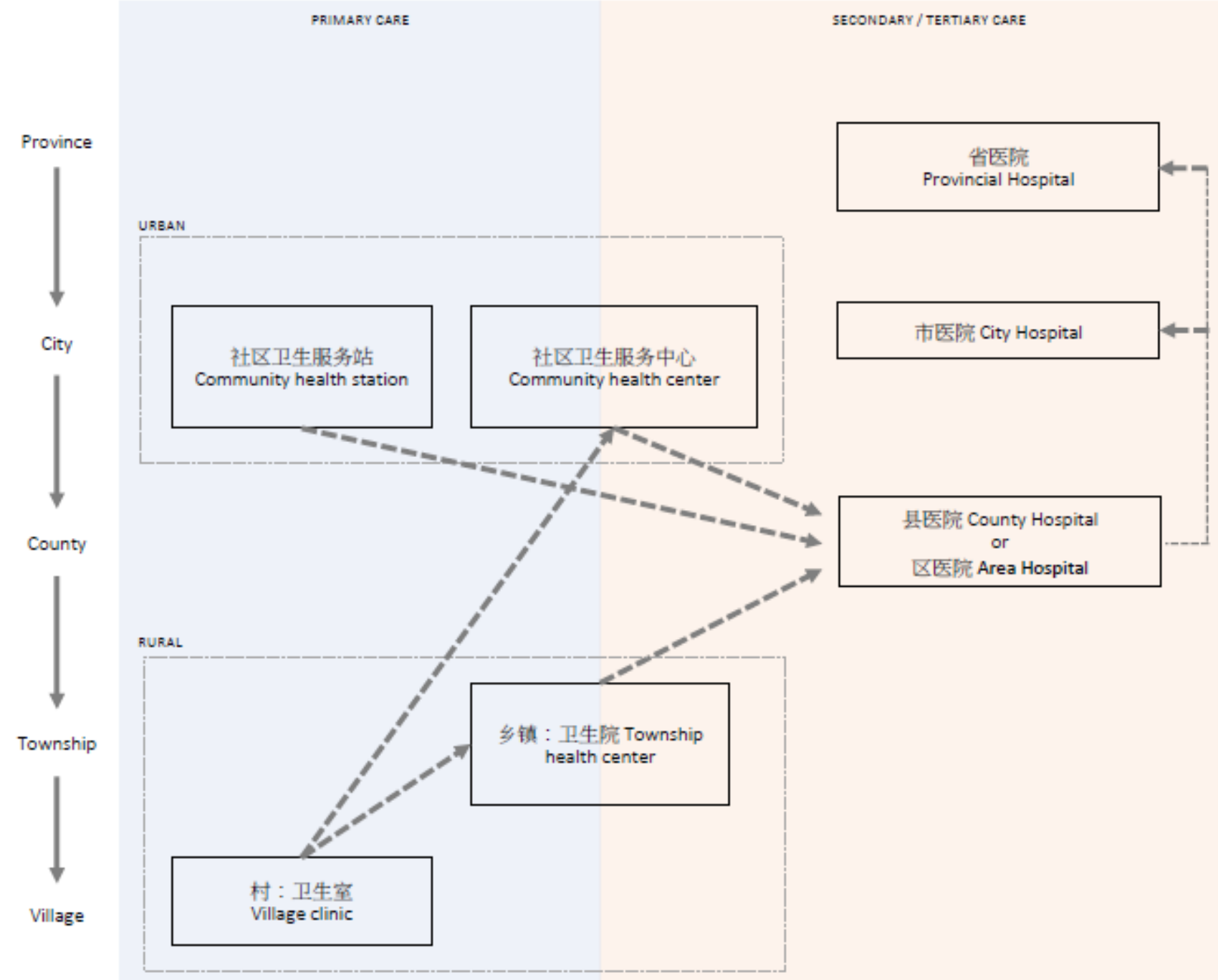

Figure 2

China's Primary Healthcare System schema by Location LEGEND: China's primary health system consists of community health stations and community health centers in urban areas, and village clinics and township health centers in rural areas. This simplified model shows that community health centers and township health centers serve both primary (blue) and secondary (orange) health care with basic hospital inpatient services. This diagram does not show the three-tiered, ten-class Chinese hospital system which is based on size of hospital, as well as specialization of medical, education, and training services 
provided. Arrows indicate that if patients are seen in a community health station which cannot adequately provide care, they can go directly to county or area-level hospitals rather than being transferred via community health centers. 